\title{
Preface Issue 4-2014
}

\author{
Hans-Christoph Grunau
}

(C) Deutsche Mathematiker-Vereinigung and Springer-Verlag Berlin Heidelberg 2014

The last few years have been a good time for solving long standing geometricaltopological conjectures. This issue reports on the solution of the Willmore conjecture-the "best" topological torus is a "real" torus with ratio of radii equal to $\sqrt{2}-$ and one of Thurston's conjectures-every hyperbolic 3-manifold can be fibered over a circle, up to passing to a finite cover.

Starting in the 1960s, Thomas Willmore studied the integral of the squared mean curvature of surfaces in $\mathbb{R}^{3}$ as the simplest but most interesting frame invariant elastic bending energy. This energy had shown up already in the early 19th century-too early for a rigorous investigation. Willmore asked: What is the shape of a compact surface of fixed genus minimising the Willmore energy in this class? (In the 1990s, existence of minimisers was proved by Leon Simon, with a contribution by Matthias Bauer and Ernst Kuwert.) Willmore already knew that the genus-0-minimiser is a sphere. Assuming that symmetric surfaces require less energy than asymmetric ones (which has not been proved, yet) he studied families of geometric tori with the smaller radius 1 fixed and found that the larger radius $\sqrt{2}$ would yield the minimum in this very special family. He conjectured that this particular torus would be the genus1-minimiser. Almost 50 years later Fernando Marques and André Neves found and published a 100-page-proof. In the present survey article, disregarding technical details, they explain their approach and their fundamental ideas and provide also an excellent historical and mathematical background.

After Thurston's Geometrization Conjecture was finally solved about 10 years ago by Grigori Perelman, one of the most important questions in three-dimensional topology has been to understand the hyperbolic 3-manifolds. William Thurston's 1982-

\footnotetext{
H.-Ch. Grunau ( $\bowtie)$

Institut für Analysis und Numerik, Fakultät für Mathematik, Otto-von-Guericke-Universität, Postfach 4120, 39016 Magdeburg, Germany

e-mail: hans-christoph.grunau@ovgu.de
} 
paper "Three-dimensional manifolds, Kleinian groups and hyperbolic geometry" (see the "Classics Revisited"-contribution of this year's first issue) lists 24 at that time open problems in 3-manifolds-theory. Problem No. 18 reads as follows: "Does every finite volume hyperbolic 3-manifold have a finite cover which fibers over the circle?" This conjecture was indeed proved 2012 by Ian Agol and Dani Wise. Stefan Friedl explains their work and the requisite background in his very comprehensible survey article "Thurston's Vision and the Virtual Fibering Theorem for 3-Manifolds." As Stefan Friedl explains, after Agol \& Wise, only "Thurston's last challenge" concerning ratios of volumes of hyperbolic 3-manifolds remains to be solved.

In this issue the "Classics Revisited"-contribution (in collaboration with the Zentralblatt für Mathematik) is written by Jean Mawhin. It concerns the "Cours d'Analyse Infinitésimale" of Charles-Jean de La Vallée Poussin, who is still famous for his proof (independent of Hadamard's) of the prime number theorem. These textbooks were used in Belgium for an exceptionally long time. Reviewers praise the originality, clearness and elegance of the exposition and its merits as a handbook in analysis.

In the "Book Reviews"- section Günter M. Ziegler gives us a critical view of Alexander Soifer's “The Mathematical Coloring Book". Johannes Huebschmann evaluates the book on "Poisson Structures" written by Camille Laurent-Gengoux, Anne Picherau, and Pol Vanhaecke. 